\title{
Update on Contact Dermatitis and Patch Testing in Patients With Skin of Color
}

\author{
Imara Scott, MD; Amber Reck Atwater, MD; Margo Reeder, MD
}

\section{PRACTICE POINTS}

- Similar rates of allergic contact dermatitis (ACD) exist between Black and White patients, with some differences in allergen profiles.

- Patch testing in patients with skin of color (SOC) may require side lighting and palpation, as erythema may be absent or minimal.

- Dermatologic training in evaluation and management of patients with $\mathrm{SOC}$ and $\mathrm{ACD}$ is vital.

- Distance to clinic and county poverty rate may adversely affect timely referral to a contact dermatitis specialist.
Allergic contact dermatitis (ACD) is a common dermatologic condition that presents with unique features in patients with skin of color (SOC). Additionally, the types of allergens that most frequently cause ACD vary in Black and White patients and are attributed to cultural differences between these 2 groups. Similarly, accurate patch testing in the SOC demographic requires an understanding of the morphologic differences between a positive patch test result in darker skin types compared to lighter skin types. Knowledge of these differences is essential to effectively diagnose and evaluate patients with ACD. There are identifiable disparities in duration of dermatitis affecting patients with ACD who live more than 50 miles from a patch testing center as well as those who live in impoverished areas. Dermatologists should be aware of these factors that impact patient care in appreciable ways so they are better prepared to serve these populations.

Cutis. 2021;108:10-12.
7 he world is an increasingly diverse place, which has particular relevance for the dermatologist. Skin color plays a significant role in diagnostic approach, as there are important differences in how cutaneous disease presents in patients with skin of color (SOC). Therefore, education about these differences is imperative. In this review, we focus on allergic contact dermatitis (ACD) and patch testing in patients with SOC. We discuss allergens common to this demographic and challenges encountered in patch testing patients with SOC. We also identify key health care disparities in the evaluation and management of ACD in this population.

\section{Has contact allergy in SOC populations been studied in North America?}

Over the last 2 decades, there have been only a handful of North American studies that address contact allergy in SOC populations. Patch test results from 114 Black patients and 877 White patients at the Cleveland Clinic from 1988 to 1991 showed that overall allergy frequency was relatively similar (43.0\% vs $43.6 \%$ ). There were notable differences in allergen sensitization. Paraphenylenediamine (PPD), which is used in hair dye, had more positive patch test reactions in Black patients $(10.6 \%$ vs $4.5 \%)$, and both PPD (21.2\% vs $4.2 \%)$ and imidazolidinyl urea, a formaldehyde-releasing preservative $(9.1 \%$ vs $2.6 \%)$, were more frequently allergenic in Black men compared to White men. ${ }^{1}$ Patch test results from the North American Contact Dermatitis Group from 1992 to 1998 described similar results, with minimal variation in the prevalence of ACD among 1014 Black and

Drs. Scott and Atwater are from the Department of Dermatology, Duke University School of Medicine, Durham, North Carolina. Dr. Reeder is from the Department of Dermatology, University of Wisconsin School of Medicine and Public Health, Madison.

Drs. Scott and Reeder report no conflict of interest. Dr. Atwater is Immediate Past President of the American Contact Dermatitis Society (ACDS). Correspondence: Amber Reck Atwater, MD (atwat012@gmail.com).

doi:10.12788/cutis.0292 
8610 White patients $(47 \%-49 \%$ vs $46 \%-49 \%) .{ }^{2}$ Positive patch test reactions to PPD were higher in Black patients for 2 of 3 test cycles (13.5\% vs 5.8\% [1994-1996] and $10.3 \%$ vs $5.3 \%$ [1996-1998]). Positive patch test reactions were higher in White patients for dimethylol dimethyl hydantoin, a formaldehyde-releasing preservative, also for 2 of 3 test cycles (1.8\% vs 0\% [1992-1994] and 2.8\% vs $0.3 \%$ [1994-1996]). Finally, positive patch test reactions to thioureas (rubber accelerators) had a mixed picture: 2 test cycles were higher in Black patients $(1.9 \%$ vs $1.0 \%$ [1992-1994] and $1.3 \%$ vs $0.7 \%$ [1994-1996]), but the third cycle (1996-1998) was lower (0.7\% vs $1.4 \%)$. Positive patch test reactions to the metal cobalt chloride were higher in Black patients in just 1 test cycle $(9.2 \%$ vs $6.6 \%$ [1992-1994]). The authors suggested that the use of darker hair dyes in the Black community may lead to more sensitization to PPD. They also theorized that this population's more frequent use of ointment-based skin care products may make them less susceptible to sensitization to preservatives such as formaldehyde, which more commonly are found in water-based products such as creams. They concluded that differences in sensitization patterns likely were driven by cultural practices affecting exposures. ${ }^{2}$

In 2016, the North American Contact Dermatitis Group reported patch test results in 434 Black and 6634 White patients (1998-2006). ${ }^{3}$ Again, ACD prevalence was about the same in both groups ( $45.9 \%$ vs $43.6 \%$ ). However, they reported several allergens with different reaction patterns. Black patients had higher risk ratios (RRs) for 3 rubber accelerators: mercaptobenzothiazole (RR, 2.10), mercapto mix (RR, 2.27), and thiuram mix (RR, 1.44). They also reacted to PPD (RR, 1.56) and the antibiotic bacitracin (RR, 1.34) at higher frequencies than White patients, who more frequently reacted to formaldehyde (RR, 0.58); the formaldehyde-releasing preservatives quaternium-15 (RR, 0.63) and diazolidinyl urea (in petrolatum: RR, 0.44; aqueous: RR, 0.47 ); the clothing finish ethylene urea melamine formalin resin (RR, 0.45); and the fragrances fragrance mix $1(R R, 0.65)$ and balsam of Peru (RR, 0.55).

Patch testing of 139 African American or Black patients at the Cleveland Clinic (2003-2012) revealed that this population most commonly had positive reactions to nickel $(27.5 \%)$, fragrance mix (18.1\%), bacitracin $(13.0 \%)$, balsam of Peru (12.3\%), and PPD (10.9\%). The authors highlighted unique features of physical examination in patients with darker skin types, including lichenification and/or hyperpigmentation in those with ACD and the potential for lack of erythema and/or a papular reaction with patch test readings. ${ }^{4}$ Recently, data was presented at the American Contact Dermatitis Society Annual Meeting (March 2021) on patterns of ACD in Black and White patch tested patients in Philadelphia (2009-2019). ${ }^{5}$ Using the North American 80 comprehensive series, the researchers documented statistically significant differences in allergen sensitivity between the 2 groups. Black patients reacted to disperse blue dye $(P=.019)$ and textile dye mix $(P=.001)$ at higher frequencies. There was a nonsignificant trend of more frequent positive reactions to PPD in Black patients (11\% vs $6 \%){ }^{5}$

Notably, all of these studies examined only 1 or 2 racial groups with a focus on Black patients. Some authors commented that this was due to low numbers of Hispanic, Asian and Pacific Islander, and Native American patients in tested populations. ${ }^{2,3,5}$ With approximately $13 \%$ of the US population self-identifying as Black, ${ }^{6}$ these patients and other minority races typically are underrepresented in large patch test studies. More data on patch test results for these groups is necessary for a complete understanding of patch testing in patients with SOC.

\section{What are the challenges in patch testing SOC populations?}

Patch testing in patients with SOC requires additional skills and experience. Darker skin does not reveal erythema as strikingly as lighter skin, making it more difficult to appreciate subtle color changes. Moreover, multiple studies have shown that ACD can have different presentations in Black patients. ${ }^{4,7,8}$ Lichenification and hyperpigmentation may be early signs of ACD in comparison to bright erythema and vesicles that can be seen in lighter skin types. It also has been reported that scalp ACD can be mistaken for seborrheic dermatitis due to lack of erythema. ${ }^{7}$ Without a high degree of clinical suspicion, a diagnosis of ACD can be missed in this patient population.

Patch test interpretation also can be challenging in patients with SOC. An early papular or follicular eruption with minimal erythema can signal a positive reaction. ${ }^{4,7}$ Because of these potentially subtle changes, patch testers should exercise care and attention when reading results for SOC populations. We recommend ample side lighting, palpation for adequate identification of positive reactions, and double-checking for positives that may have been overlooked on the initial review of findings. ${ }^{4,7}$

\section{What health care disparities impact the evalua- tion and management of ACD?}

There are many factors at play in this dialogue. The challenges we identified in diagnosing ACD in darker skin types are important to consider. Lack of familiarity with these unique features can lead to a delay in diagnosis and ultimately a delay in referral for patch testing. This is where dermatology training can help fill in the gap, but are the majority of programs equipped to do so? Inadequate education and exposure to patients with SOC is an issue for many dermatology residency programs. Surveys of residents and program directors in geographically less diverse regions may not receive adequate education or exposure to patients with SOC. ${ }^{9}$ Further, there is a lack of representation of SOC images for general dermatologic conditions in textbooks, ${ }^{10,11}$ which has a profound impact on the dermatologist's ability to recognize 
common diseases in darker skin types. A 2019 survey of more than 5000 images from 2 dermatology textbooks showed SOC images comprised $22 \%$ to $32 \%$ of the total images. ${ }^{11}$ However, SOC images are overrepresented in textbooks for sexually transmitted infections, constituting $47 \%$ to $58 \%$ of the images; they made up $28 \%$ of images for nonvenereal infections. ${ }^{11}$ Why is that? In this article, we have shown the prevalence of ACD to be nearly equivalent in Black and White patients, yet a perusal of $\mathrm{ACD}$ images in dermatology textbooks will tell a different story. This trend deserves our attention; perhaps it is highlighting patterns of systemic racism seen in medicine. If our primary teaching materials are perpetuating stereotypes, we must consider the impact this can have on our personal implicit biases and the health care disparities that can ensue.

Additional factors impact time to diagnosis of $\mathrm{ACD}$ and referral for patch testing. A retrospective study examining distance to a North Carolina patch test referral clinic showed that patients living further from the clinic experienced a longer duration of dermatitis prior to patch test consultation and tended to live in areas with a higher county poverty rate..$^{12}$ Specifically, a $17.9 \%$ increase $(P<.001)$ in the median duration of dermatitis was observed for every 50 -mile increase in distance to the patch test clinic. County poverty rate was measured by the percentage of residents living below the poverty threshold; for every $5 \%$ increase in county poverty rate, a $16.3 \%$ increase $(P<.032)$ in duration of dermatitis was found. ${ }^{12}$

These data highlight a relationship with which many dermatologists are familiar and underscore a need for dermatologists to practice in areas that are more geographically accessible. The recently increased utilization of telehealth modalities can potentially help to bridge this gap by decreasing delays in diagnosis and providing more affordable options for evaluation by a dermatologist for patients with socioeconomic obstacles.

\section{Final Interpretation}

The prevalence of ACD among Black and White patients is similar; however, there are important differences in patch test reaction frequencies that may be related to the diverse exposure patterns for each group. Additionally, patients with SOC may have unique clinical presentations of ACD, such as lichenification and hyperpigmentation. Darker skin types also may require specialized techniques for accurate patch test readings. It is imperative that dermatologists are trained to recognize all of these features. Health care disparities come in many forms and, in this setting, can result in delayed referral for patch testing. Additional studies are needed to further examine these health care disparities and identify potential solutions.

\section{REFERENCES}

1. Dickel H, Taylor JS, Evey P, et al. Comparison of patch test results with a standard series among white and black racial groups. Am J Contact Dermat. 2001;12:77-82.

2. Deleo VA, Taylor SC, Belsito DV, et al. The effect of race and ethnicity on patch test results. J Am Acad Dermatol. 2002;46 (2 suppl understanding):S107-S112.

3. Deleo VA, Alexis A, Warshaw EM, et al. The association of race lethnicity and patch test results: North American Contact Dermatitis Group, 1998-2006. Dermatitis. 2016;27:288-292.

4. Yu SH, Khanna U, Taylor JS, et al. Patch testing in the African American population: a 10-year experience. Dermatitis. 2019;30:277-278.

5. GargVS, Zhan, T, Brod B, et al. Patterns of allergic contact dermatitis in African Americans and Caucasians in a major metropolitan area over a ten-year period. Presented at: 32nd American Contact Dermatitis Society Annual Meeting (virtual); March 17-18, 2021.

6. United States Census Bureau. QuickFacts-United States. Accessed June 11, 2021. https://www.census.gov/quickfacts/fact/table /US/PST045219

7. Stallings A, Sood A. Hair-care practices in African American women: potential for allergic contact dermatitis. Semin Cutan Med Surg. 2016;35:207-210

8. Otrofanowei E, Ayanlowo OO, Akinkugbe A, et al. Clinico-etiologic profile of hand dermatitis and patch response of patients at a tertiary hospital in Lagos, Nigeria: results of a prospective observational study. Int J Dermatol. 2018;57:149-155.

9. Nijhawan RI, Jacob SE, Woolery-Lloyd H. Skin of color education in dermatology residency programs: does residency training reflect the changing demographics of the United States? I Am Acad Dermatol. 2008;59:615-618.

10. Ebede T, Papier A. Disparities in dermatology educational resources. J Am Acad Dermatol. 2006;55:687-690.

11. Lester JC, Taylor SC, Chren MM. Under-representation of skin of colour in dermatology images: not just an educational issue. $\mathrm{Br} J$ Dermatol. 2019;180:1521-1522.

12. Rodriguez-Homs LG, Liu B, Green CL, et al. Duration of dermatitis before patch test appointment is associated with distance to clinic and county poverty rate. Dermatitis. 2020;31:259-264. 